\title{
Dysregulated calcium homeostasis prevents plasma membrane repair in Anoctamin 5/TMEM16E-deficient patient muscle cells
}

\author{
Goutam Chandra', Aurelia Defour ${ }^{1,7}$, Kamel Mamchoui ${ }^{2}$, Kalpana Pandey $\mathbb{1}^{3}$, Soumya Mishra', Vincent Mouly ${ }^{2}$, \\ SenChandra Sreetama', Mohammad Mahad Ahmad', Ibrahim Mahjneh4, Hiroki Morizono (1) ${ }^{1,5}$,

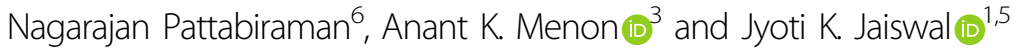

\begin{abstract}
Autosomal recessive mutations in Anoctamin 5 (ANO5/TMEM16E), a member of the transmembrane 16 (TMEM16) family of $\mathrm{Ca}^{2+}$-activated ion channels and phospholipid scramblases, cause adult-onset muscular dystrophies (limb girdle muscular dystrophy 2L (LGMD2L) and Miyoshi Muscular Dystrophy (MMD3). However, the molecular role of ANO5 is unclear and ANO5 knockout mouse models show conflicting requirements of ANO5 in muscle. To study the role of ANO5 in human muscle cells we generated a myoblast line from a MMD3-patient carrying the c.2272C $>$ T mutation, which we find causes the mutant protein to be degraded. The patient myoblasts exhibit normal myogenesis, but are compromised in their plasma membrane repair (PMR) ability. The repair deficit is linked to the poor ability of the endoplasmic reticulum (ER) to clear cytosolic $\mathrm{Ca}^{2+}$ increase caused by focal plasma membrane injury. Expression of wild-type ANO5 or pharmacological prevention of injury-triggered cytosolic $\mathrm{Ca}^{2+}$ overload enable injured patient muscle cells to repair. A homology model of ANO5 shows that several of the known LGMD2L/MMD3 patient mutations line the transmembrane region of the protein implicated in its channel activity. These results point to a role of cytosolic $\mathrm{Ca}^{2+}$ homeostasis in PMR, indicate a role for ANO5 in ER-mediated cytosolic $\mathrm{Ca}^{2+}$ uptake and identify normalization of cytosolic $\mathrm{Ca}^{2+}$ homeostasis as a potential therapeutic approach to treat muscular dystrophies caused by ANO5 deficit.
\end{abstract}

\section{Introduction}

Mutations in the Anoctamin 5 (ANO5)/ TransMEMbrane $16 E$ (TMEM16E) gene underlie the most common adult onset recessive muscular dystrophy in Northern Europe $^{1-3}$. This includes limb-girdle muscular dystrophy type 2L (LGMD 2L) and Miyoshi myopathy type 3 (MMD3), which are characterized by high levels of serum

\footnotetext{
Correspondence: Jyoti K. Jaiswal (jkjaiswal@cnmc.org)

${ }^{1}$ Center of Genetic Medicine Research, Children's National Health System, 111 Michigan Avenue, NW, Washington, DC 20010, USA

${ }^{2}$ Center for Research in Myology, Sorbonne Universités, UPMC Université Paris 06, INSERM UMRS974, 47 Boulevard de l'hôpital, 75013 Paris, France Full list of author information is available at the end of the article. Edited by I. Amelio
}

creatine kinase (CK) indicating myofiber damage, exercise-induced muscle pain, and rare presentation of cardiomyopathy or respiratory failure. LGMD $2 \mathrm{~L}$ causes proximal, asymmetric weakness of quadriceps, femoris and biceps brachii, and minimal weakness of distal lower limbs. MMD3 results in weakness of the calf and other distal muscles, making it difficult for patients to walk on their toes, which progresses and results in asymmetric weakness of proximal limb-girdle muscles ${ }^{4}$. ANO5/ TMEM16E gene mutations also result in the autosomal dominant bone disorder gnathodiaphyseal dysplasia (GDD). Thus, another name for $A N O 5$ is $G D D 1^{5}$. ANO5/ TMEM16E/GDD1 (henceforth ANO5) belongs to the

\section{(c) The Author(s) 2019}

(c) (i) Open Access This article is licensed under a Creative Commons Attribution 4.0 International License, which permits use, sharing, adaptation, distribution and reproduction cc) in any medium or format, as long as you give appropriate credit to the original author(s) and the source, provide a link to the Creative Commons license, and indicate if changes were made. The images or other third party material in this article are included in the article's Creative Commons license, unless indicated otherwise in a credit line to the material. If material is not included in the article's Creative Commons license and your intended use is not permitted by statutory regulation or exceeds the permitted use, you will need to obtain permission directly from the copyright holder. To view a copy of this license, visit http://creativecommons.org/licenses/by/4.0/. 
ten-member human Anoctamin/TMEM16 membrane protein family that includes $\mathrm{Ca}^{2+}$-activated ion channels (ANO1/TMEM16A, ANO2/TMEM16B) and phospholipid scramblases (ANO3, ANO4, ANO6, ANO7, and ANO10 $)^{6,7}$. In reconstitution-based assays ANO6/ TMEM16F and its fungal homologues Aspergillus fumigatus TMEM16 (afTMEM16) and Nectria haematococca TMEM16 (nhTMEM16) show both scramblase and relatively unselective ion channel activity that are regulated by $\mathrm{Ca}^{2+8-16}$.

ANO5 is expressed primarily in muscle and bone, localizing to the endoplasmic reticulum $(E R)^{17-19}$. Evidence exists in favor and against its ability to execute canonical functions of the Anoctamin/TMEM16 family, i.e. to act as a $\mathrm{Ca}^{2+}$-activated $\mathrm{Cl}^{-}$channel and/or a phospholipid scramblase ${ }^{19-25}$. It is likely that similar to ANO6/TMEM16F, ANO5 is also a dual function ion channel and phospholipid scramblase. Thus, transplantation of a short stretch of amino acids (the 'scrambling domain' or SCRD) from ANO5 onto ANO1/TMEM16A, imparts $\mathrm{Ca}^{2+}$-activated phospholipid scramblase activity to this $\mathrm{Cl}^{-}$channel ${ }^{21,26}$. Heterologous overexpression of ANO5 in the plasma membrane of HEK293 cells associates with $\mathrm{Ca}^{2+}$-dependent phospholipid scrambling and non-selective ion currents ${ }^{22,26}$.

The physiological role of ANO5 remains elusive, despite the large number of known disease-associated ANO5 mutations $^{27}$. In mouse, ANO5 is implicated in muscle differentiation and membrane repair ${ }^{17,28}$, while our studies with MMD3 patient fibroblasts revealed a deficiency in PMR ability which correlated with the patient's clinical symptoms including elevated serum CK level, and muscle weakness and pain ${ }^{29}$. Consistent with this, electron microscopic analysis of patient muscle showed sarcolemmal disruptions ${ }^{4,30}$. Thus, $\mathrm{Ca}^{2+}$-dependent PMR in human muscle cells may require ANO5. Such a role has been established for dysferlin protein, the lack of which causes distinct dystrophies termed LGMD2B and Miyoshi myopathy $^{31,32}$. However, unlike dysferlin-deficient patient muscle cells, where PMR is compromised by poor lysosomal exocytosis ${ }^{33}$, ANO5-deficient patient fibroblasts displayed normal injury-triggered lysosomal exocytosis ${ }^{29}$. Furthermore, ANO5 overexpression did not rescue the muscle pathology of dysferlin-deficient mice ${ }^{34}$. Thus, the basis of defective PMR in ANO5-deficient human fibroblasts is distinct from that caused by dysferlin deficiency.

Pathologies noted in ANO5-deficient patients have not been uniformly recapitulated in ANO5 knockout animal models ${ }^{19,28,35}$. For example, muscle pathology and high serum CK, with in vivo and in vitro deficits in myogenic differentiation and ex vivo PMR deficit were noted in only one of several described ANO5 knockout mouse models $^{19,28,35}$, whereas a recently described ANO5 knockout rabbit model demonstrated progressive muscle histological damage ${ }^{36}$. Thus, genetic background, disease model-specific differences and knockout strategy may affect disease manifestation. These conflicting data highlight the need to examine the effect of ANO5 mutations directly by using patient muscle cell-based models. We now report the first patient-derived myoblast cell model for ANO5 deficit. We find that ANO5 is dispensable for myogenic differentiation of the human muscle cells, but is required for the repair of injured muscle cells by facilitating cytosolic $\mathrm{Ca}^{2+}$ homeostasis.

\section{Results}

ANO5 patient muscle cells grow and differentiate normally

PMR and myogenesis are cellular deficits reported in LGMD2L patient and animal models ${ }^{28,29,36}$. To understand the basis for this pathology, we generated a stable myoblast cell line from a MMD3 patient carrying a homozygous mutation (c.2272C>T) in the ANO5 gene (Fig. 1a), and assessed the growth and differentiation ability of these cells. Growth of patient and healthy myoblasts was indistinguishable (doubling time 18.1 \pm $1.1 \mathrm{~h}$ vs $16.9 \pm 1.1 \mathrm{~h}$, respectively). Immunostaining showed significantly reduced levels of ANO5 in the patient myoblasts compared with healthy cells (Fig. 1b, c). Quantitative RT-PCR showed that ANO5 mRNA levels were unaffected (Fig. 1d), suggesting that the c.2272C $>\mathrm{T}$ mutation (replacement of Arginine 758 with Cysteine) causes degradation of the ANO5 protein.

Due to previous reports of decreased myogenic differentiation between the cultured WT and ANO5 deficient mouse myoblasts, we examined the ability of the patient myoblasts to undergo differentiation by allowing confluent cultures of myoblasts to differentiate for 10 days. Extent of myoblast fusion, monitored by counting myonuclei in myosin heavy chain 3 (MYH3)-positive myotubes, was similar in patient and healthy myotubes (Fig. 1e, f). Western Blot analysis of the differentiation marker proteins - MYH3, desmin, and $\alpha$-actinin identified that while the expression levels of these proteins showed minor variations between healthy and patient myoblasts, the overall level and timing of induction of these markers was not reduced in the patient myoblasts (Fig. 1g-j). Above findings showed that unlike the ANO5 deficient mouse cells, MMD3 patient myoblasts do not exhibit any detectable deficit in their growth and differentiation.

\section{ANO5-deficient patient myoblasts show poor plasma membrane repair}

Analysis of fibroblasts from MMD3 patients with the c. $2272 \mathrm{C}>\mathrm{T} / \mathrm{R} 758 \mathrm{C}$ mutation reveal that they also lack ANO5 protein (Supplemental Fig. 1a, b), and, as we reported previously ${ }^{29}$, they show poor PMR following focal injury (Supplemental Fig. 1c-e) or laser injury (Supplemental Fig. 1f, g). We therefore examined if 


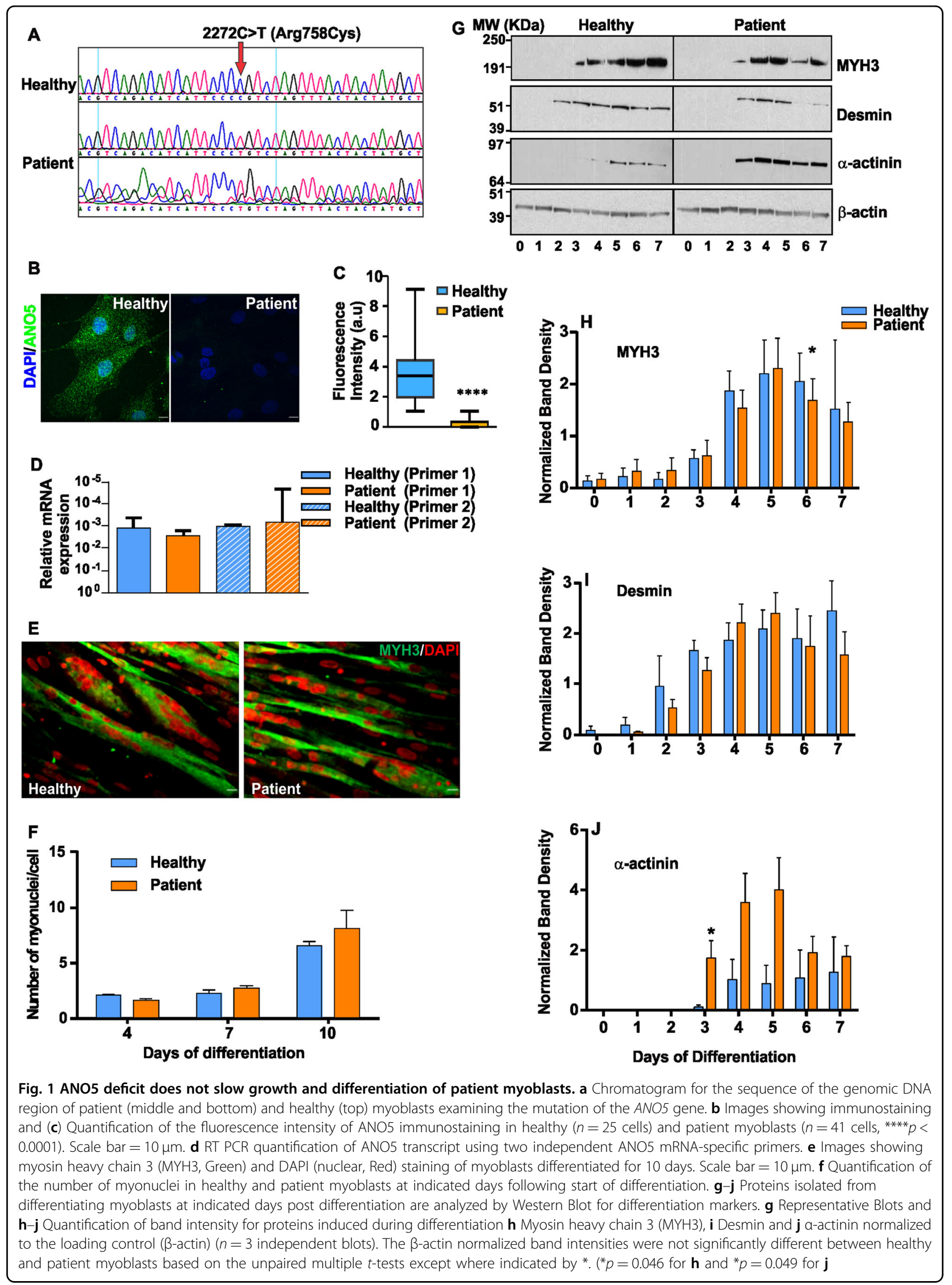


myoblasts from our patient have a reduced ability to repair their plasma membrane after injury. First, we used a glass bead injury assay ${ }^{33,37}$ - myoblasts injured by rolling glass-beads are labeled with green fluoresceindextran; subsequent labeling with red rhodamine dextran results in predominantly red labeling of injured cells that fail to repair. A significantly greater number of glass beadinjured patient cells were labeled red as compared to the corresponding healthy myoblasts (Fig. 2a, b). Thus, ANO5 deficit also causes poor repair of patient muscle cells. As an independent test of membrane repair ability, we used a laser injury assay ${ }^{33,37}$, where injury causes the lipophilic FM dye to enter the cell until the entry is prevented within a minute by PMR. In patient myoblasts, FM-dye entry continued beyond $2 \mathrm{~min}$ post-injury (Fig. 2c-e, Supplemental Fig. 2a (trace labeled 'Patient')), indicating poor PMR.

We next tested if ANO5 expression could rescue the PMR deficit of patient cells. We used transient transfection to express ANO5-GFP in patient cells (Fig. 2c bottom panel and inset) and performed the laser injury assay using FM4-64 dye. ANO5-GFP expression ameliorated the poor repair phenotype of MMD3 patient myoblasts: their ability to exclude dye entry following focal injury became similar to that of healthy cells (Fig. $2 \mathrm{c}-\mathrm{e})$. In contrast to ANO5, expressing dysferlin, which rescues the repair deficit in LGMD2B cells, did not reverse the PMR defect in patient myoblasts (Supplemental Fig. 2a). These results with two different patient cell lineages (myoblasts and fibroblasts) and rescue by re-expression of ANO5 establish the requirement of ANO5 for cell membrane repair in MMD3 patients. The results also indicate that unlike GFP fusions that affect the activity of other TMEM16 proteins ${ }^{15}$, the ANO5GFP fusion protein is functional. Further, as can be expected $^{33,34}$ ANO5 plays a role distinct from that of dysferlin in membrane repair.

ANO5 has been localized to the ER in a variety of nonmuscle cells ${ }^{18}$. As ANO5-GFP is functional in terms of its ability to rescue the membrane-repair phenotype of patient myoblasts (Fig. 2c-e), we used it to investigate ANO5 localization in a human cell line (HER-911). ANO5-GFP localized to the ER in these cells (Supplemental Fig. 2b). Similarly, co-expression of ANO5-GFP with an RFP-tagged ER luminal marker (RFP-KDEL or ER-RFP) in healthy human myoblasts showed overlapping reticular staining of both proteins, confirming ER localization of ANO5 in human myoblasts (Fig. 2f). Fluorescence recovery after photobleaching measurements of ER membrane-localized membrane proteins Sec61-mCherry, ANO5-GFP showed similar recovery kinetics indicating that ANO5-GFP localizes to the ER membrane and is not trapped in the ER due to misfolding or other potential artefacts (Fig. 2g, h).

\section{ANO5 accumulates at site of repair and helps maintain ER structural integrity}

To study the involvement of ANO5 in PMR, we imaged ANO5-GFP and the luminal ER marker RFP-KDEL in healthy human myoblasts following focal laser injury. Interestingly, ANO5-GFP selectively accumulated at the site of injury, even as the ER at the injury site appeared to fragment, as indicated by the luminal marker RFP-KDEL (Fig. 3a, b). The injury-induced loss in ER structural integrity was associated with leakage of the ER luminal marker RFP-KDEL, indicated by the decrease in RFP signal (Fig. 3b). Acute ER fragmentation is triggered by persistent increases in cytosolic calcium $\left(\left[\mathrm{Ca}^{2+}\right]_{c}\right)$, generated by exposing cells to purinergic signals or to the $\mathrm{Ca}^{2+}$ ionophore ionomycin, in the presence of extracellular $\mathrm{Ca}^{2+}{ }^{38}$. ER localized $\mathrm{Ca}^{2+}$-activated chloride channels $(\mathrm{CaCCs})$ are required for regulating cytosolic calcium in response to purinergic signaling in epithelial cells ${ }^{39}$. Considering the ER localization and $\mathrm{Ca}^{2+}$-activated non-selective ion channel activity of $\mathrm{ANO}^{22,25,26}$, we hypothesized that ANO5 may mitigate the rise in $\left(\left[\mathrm{Ca}^{2+}\right]_{\mathrm{c}}\right.$ resulting from focal injury, thus maintaining ER integrity in injured cells. Using RFP-KDEL we observed that while focal injury of healthy cells caused about $10 \%$ of the cellular ER near the injury site was fragmented. Use of ER membrane marker Sec61-mCherry independently confirmed fragmentation of the ER near the injury site (Supplemental video 3). However, in MMD3 patient myoblasts focal injury resulted in fragmentation of nearly half of all of the cellular ER (Fig. 3c, d, Supplemental videos 1,2$)$. Furthermore, total internal reflection fluorescence (TIRF) microscopy to monitor plasma membrane proximal $\mathrm{ER}^{40}$ revealed a $\sim 2$-fold reduction in RFP-KDEL signal in the vicinity of the PM in uninjured patient myoblasts compared with healthy myoblasts (Supplemental Fig. 3). Taken together, these findings suggest a role of ANO5 in maintaining the plasma membrane proximity of ER in resting cells and integrity of the ER in the injured cells, possibly by playing a role in $\left[\mathrm{Ca}^{2+}\right]_{\mathrm{c}}$ homeostasis through its ion channel activity.

\section{Modeling 3-dimensional structure of ANO5 protein}

The results above suggest that ANO5 may play a role in cellular $\mathrm{Ca}^{2+}$ homeostasis by functioning as a $\mathrm{Ca}^{2+}$-dependent ion channel in the ER. As a preliminary test of this idea we asked whether patient mutations map onto regions of ANO5 that are predicted to be involved in its transport function. As ANO5 has not been structurally characterized we first built a homology model of the protein templated on the structure of Nectria haematococca TMEM16 (nhTMEM16), an ancestral structurally defined ion channel and phospholipid scramblase of the TMEM16 protein family. We carried out a structurebased alignment of the primary sequence of ANO5 with 


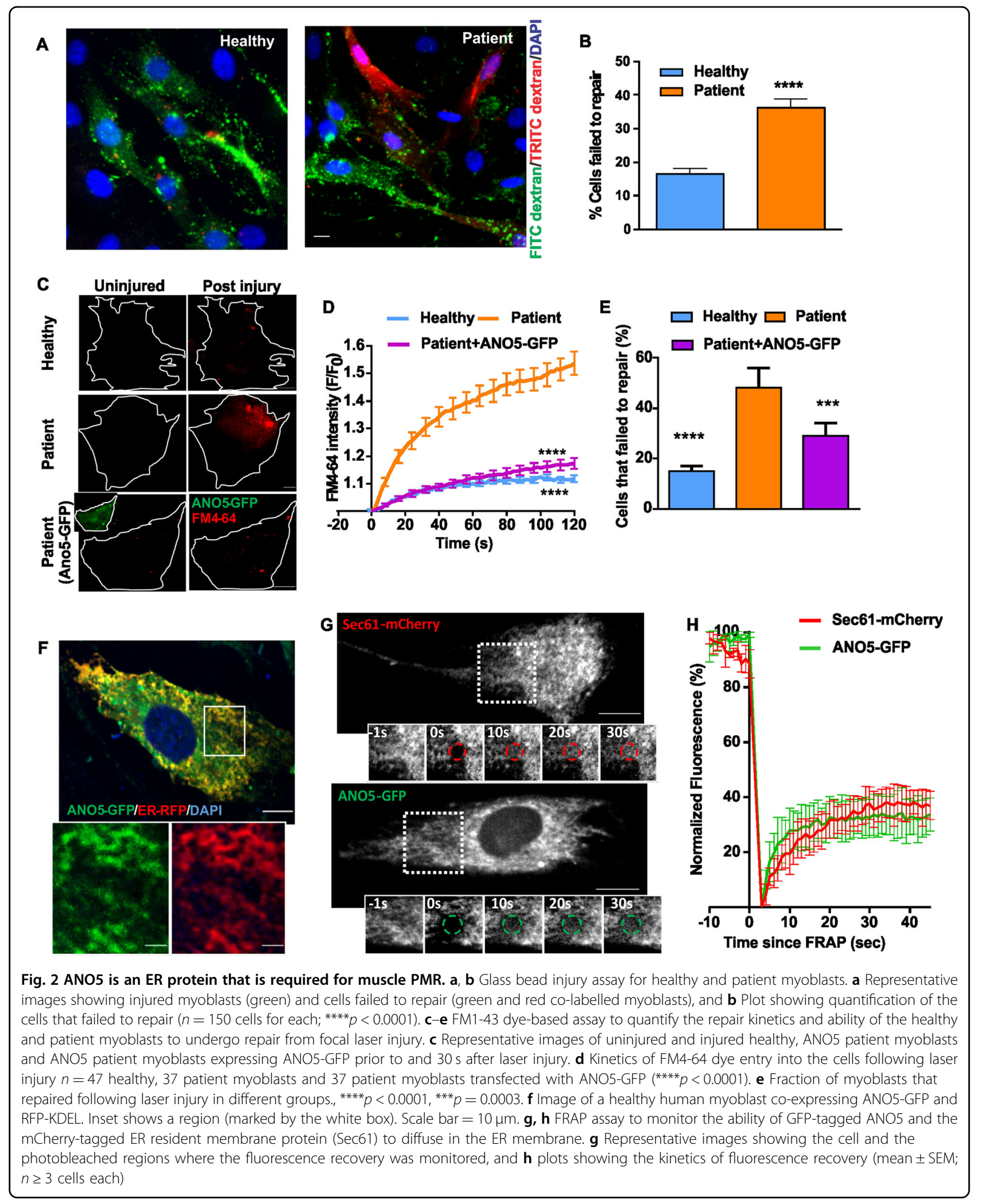


A
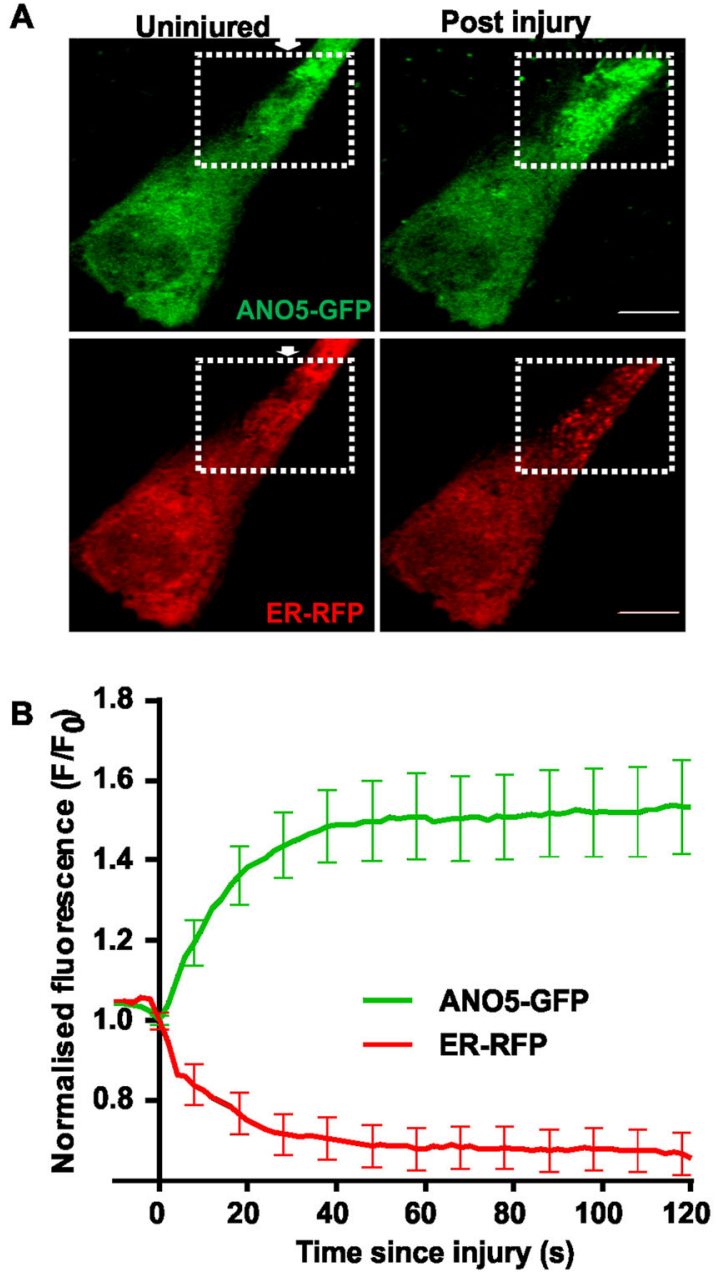

C

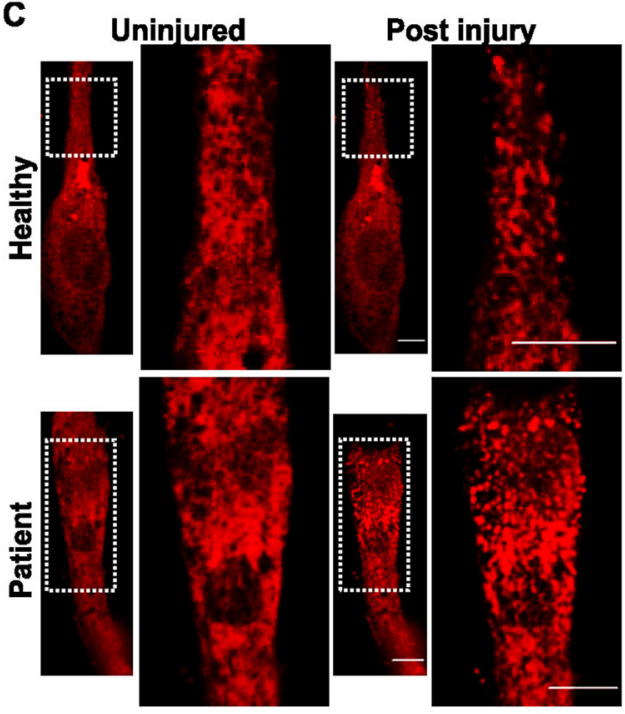

D

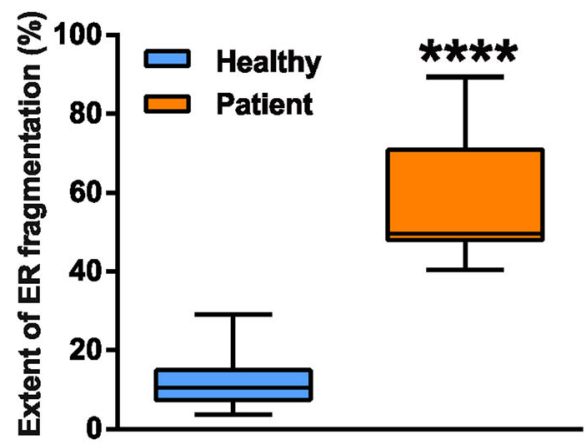

Fig. 3 Effect of injury on the ANO5 distribution and ER structure. a Images of healthy myoblasts expressing ANO5-GFP and RFP-KDEL are injured (at site marked by the arrow) by laser beam. Representative images before and $60 \mathrm{~s}$ after the injury are shown. $\mathbf{b}$ Kinetics of ANO5-GFP accumulation and RFP-KDEL loss at the site of repair (eg; region marked in panel a) is quantified in the plot. Scale bar $=10 \mu m$. $n=18$ cells $\mathbf{c}$ ANO5 patient myoblasts expressing RFP-KDEL showed significantly greater ER fragmentation (marked by the dotted box, and presented in the zoomed image). d Quantification of ER fragmentation in healthy and patient myoblasts showed that lack of ANO5 causes increased ER fragmentation following injury; $n=13$ cells (healthy) and 9 cells (Patient) $\left.{ }^{* * *} p<0.0001\right)$. Scale bar $=10 \mu \mathrm{m}$

nhTMEM16 using HHPred (Supplemental Fig. 4), and then used MODELLER to build a 3D model. The alignment located the previously identified SCRD domain as well as several other residues that are known to be important for phospholipid scrambling and ion transport activities in ANO5 and nhTMEM16 (Fig. 4a) ${ }^{14,21}$. The 3D structural model, placed in the context of the ER membrane consistent with the known localization of the protein (Fig. 4b-d), depicts ANO5 as a homodimer in which each monomer has 10 transmembrane (TM) segments (Fig. 4b). TM10 contributes significantly to the dimer interface. The $\mathrm{Ca}^{2+}$ binding site in each monomer is buried within the protein but accessible from the cytoplasmic side (shown only in the monomer on the left, Fig. $4 \mathrm{~b}$, and in more detail in Fig. 4e). The cytoplasmic C- terminal portion of the protein, as well as unstructured loops, especially a large loop between TM9 and TM10 (in the ER lumen), are not shown for simplicity. Rotation of the ANO5 dimer by $90^{\circ}$ in the plane of the membrane reveals the membrane-facing, hydrophilic transmembrane groove (subunit cavity) within each monomer that is implicated in lipid and ion transport (Fig. 4c, d; the suggested permeation pathway is shown by the dashed line in Fig. 4d). The groove is formed from elements of TM helices 3-7; it contains the SCRD on its cytoplasmic side (Fig. 4c, d, f), as well as a number of functionally important residues that control access to the transmembrane pathway from the ER lumen. These residues are noted in the sequence alignment (Fig. 4a) and their location in the $3 \mathrm{D}$ model of ANO5 protein is shown in Fig. 4f. The 


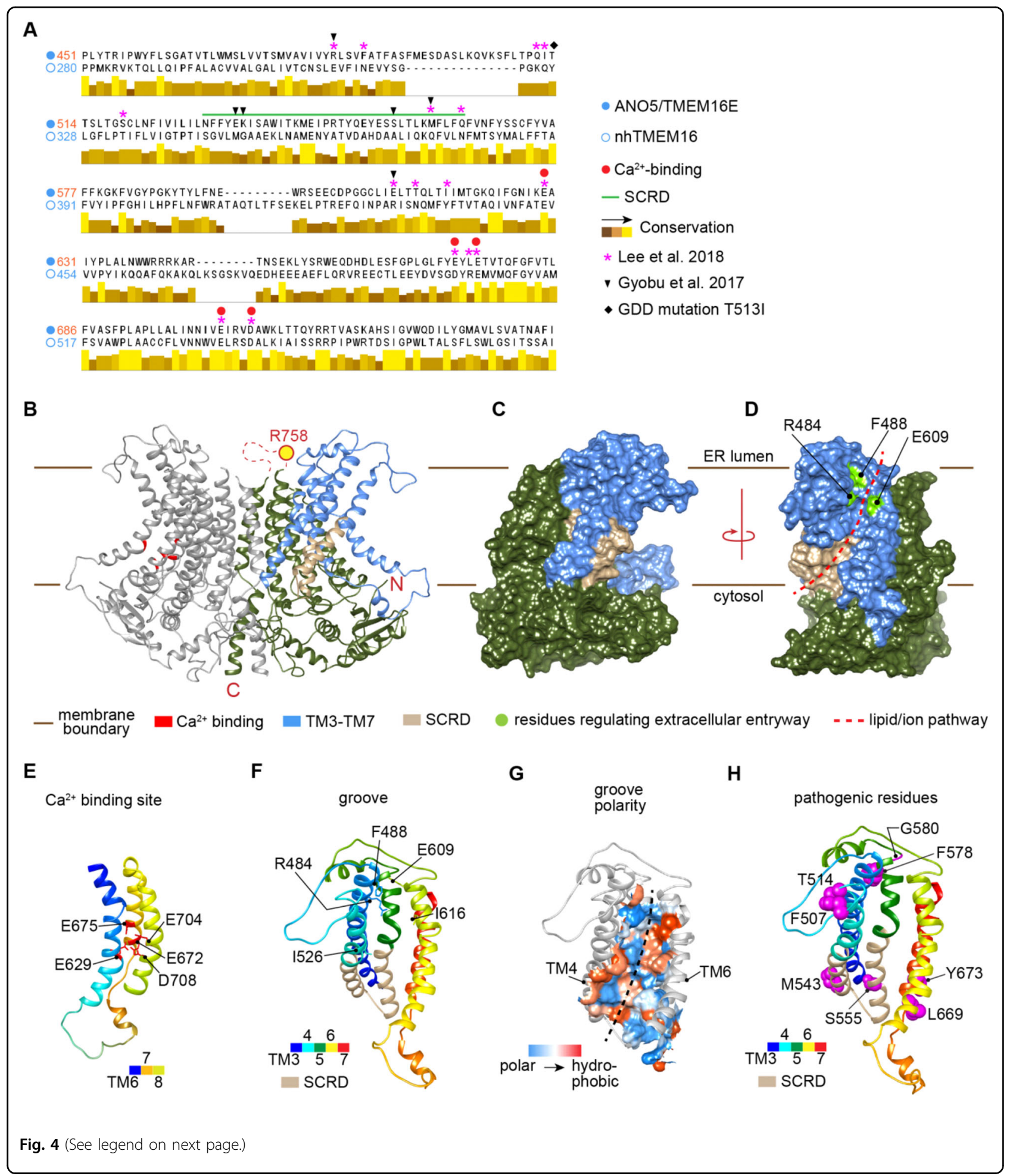

groove has a significantly polar surface consistent with its function in lipid and ion transport (Fig. 4g) but is somewhat wider in ANO5 than in nhTMEM16, consistent with the ability of ANO5 to transport the large cation Nmethyl-D-Glucamine (NMDG) ${ }^{26}$. Of interest, TM3 and
TM6 are tethered by a pair of charged residues in nhTMEM16 (E313 (TM3) and R432 (TM6)) and this feature is replicated in the 3D structural model of ANO5 (R484 (TM3) and E609 (TM6)). Disruption of the interaction between these charged residues has been proposed 
(see figure on previous page)

Fig. 4 Structural model of ANO5 based on nhTMEM structure. a Partial sequence alignment of human ANO5 and nhTMEM16. The scramblase domain (SCRD, corresponding to N530....Q564 of ANO5) is indicated with a green bar. Asterisks indicate residues in nhTMEM16 identified as being important for function ${ }^{14}$ whereas arrowheads indicate residues that were found to be important for the scramblase activity of mouse ANO5/ TMEM16E 21 . Red filled circles show residues implicated in $\mathrm{Ca}^{2+}$ binding (see also $\mathbf{b}$ and $\mathbf{e}$ ) and the black diamond indicates a residue implicated in gnathodiaphyseal dysplasia (GDD). b Homology model of ANO5 based on nhTMEM16 (PDB ID: 4WIS ${ }^{12}$ ) is shown in ribbon representation. The protein is shown as a homodimer in the plane of the ER membrane (the lumenal and cytosolic faces are indicated). Large loops on the extracellular side (notably the loop connecting TM9 and TM10) have been omitted for clarity; also omitted is the C-terminal portion on the cytoplasmic side of the protein. One monomer is shaded in light grey; the other monomer is colored in olive green with TM helices 3-7 highlighted in cornflower blue to show the portion of the protein involved in creating the transmembrane groove or subunit cavity that is implicated in ion and lipid transport across the membrane. Residues that make up the $\mathrm{Ca}^{2+}$-binding site are shown in red the monomer at left; the scramblase domain (SCRD) is shown in tan in the right monomer. For clarity, different regions are highlighted in different monomers. c Surface representation of the right monomer using the same color coding as in the ribbon representation. $\mathbf{d}-\mathbf{h}$ View of the protein after rotation by $90^{\circ}$ in the plane of the membrane. $\mathbf{d}$ Surface representation revealing the transmembrane groove and permeation pathway (dashed line). Residues that regulate lipid and ion entry on the extracellular side are indicated in chartreuse green (see also f). e Acidic residues located on TM6, 7 and 8 that make up the Ca ${ }^{2+}$-binding site are indicated. For $\mathbf{e}, \mathbf{f}$, and $\mathbf{g}$, the ribbon is color coded from N-terminal to C-terminal from blue to red via green and yellow. $\mathbf{f}$ View of the ion/lipid transporting groove. TM helices 3-7 (blue in $\mathbf{b}$ ) are shown with the SCRD domain (cytoplasmic side of TM4-TM5) colored tan; residues important for regulating access to the transport pathway from the extracellular side are indicated (see also $\mathbf{d}$ ). $\mathbf{g}$ Surface representation of the transmembrane groove of ANO5, color coded to reveal polarity. $\mathbf{h}$ View of the ANO5 groove illustrating the location of residues known to be associated with pathological phenotypes

to be part of a series of conformational changes, including rotation of TM6, that open the lipid/ion transport pathway $^{14}$ Thus, substitution of R484 and E609 with tryptophan is predicted to disrupt transport activity as seen for the corresponding $\mathrm{E} 313 \mathrm{~W}$ and $\mathrm{R} 432 \mathrm{~W}$ mutations in nhTMEM16. Many pathogenic mutations in ANO5 are found near the transmembrane groove and $\mathrm{Ca}^{2+}$ binding site as shown in (Fig. 4e, h). Thus, M543 and S555 are located within the SCRD, and Y673 is adjacent to the E672 residue implicated in $\mathrm{Ca}^{2+}$ binding. The corresponding patient mutations include M543N, S555I, S555R, and Y673C. Residues T513 and T514 are also of interest, the former being implicated in GDD while the latter is associated with adult onset muscular dystrophy. Both are at the ER lumenal end of TM4 (Fig. 4a, h).

\section{ANO5 is required for buffering the increase in cytosolic $\mathrm{Ca}^{2+}$}

The ER-localized $\mathrm{Ca}^{2+}$-activated ion channel bestrophin regulates increases in $\left[\mathrm{Ca}^{2+}\right]_{\mathrm{c}}$ caused by purinergic and other signaling pathways in epithelial cells ${ }^{39}$. We therefore examined if greater injury-triggered ER fragmentation in ANO5-deficient patient myoblasts could be due to a poor handling of elevated $\left[\mathrm{Ca}^{2+}\right]_{\mathrm{c}}$ by the patient myoblasts. We used the $\mathrm{Ca}^{2+}$-sensitive dye Fluo- 4 to measure cytosolic $\mathrm{Ca}^{2+}$ change $^{41}$ induced either by a purinergic signal (ATP) or injury. ATP induced a $\sim 20 \%$ increase in $\left[\mathrm{Ca}^{2+}\right]_{\mathrm{c}}$ in healthy cells, which returned to baseline over $15 \mathrm{~min}$. However, in patient cells ATP induced a more sustained $\left[\mathrm{Ca}^{2+}\right]_{\mathrm{c}}$ increase, followed by a slower return to baseline, resulting in $\left[\mathrm{Ca}^{2+}\right]_{\mathrm{c}}$ overload (Supplemental Fig. 5a-d). Similar to the effect seen with ATP, injury-triggered increase of $\left[\mathrm{Ca}^{2+}\right]_{\mathrm{c}}$ in the patient cells was also cleared slowly (Fig. 5a, b). While healthy myoblasts attained peak $\left[\mathrm{Ca}^{2+}\right]_{\mathrm{c}}$ levels within $10 \mathrm{~s}$ following injury, and cleared this within $40 \mathrm{~s}$ (Fig. 5a, upper panel and b, blue trace), ANO5 deficient patient myoblasts took longer $(>60 \mathrm{~s})$ to clear the $\left[\mathrm{Ca}^{2+}\right]_{\mathrm{c}}$ elevation (Fig. 5a, lower panel and b, red trace). Thus, dysregulation of $\mathrm{Ca}^{2+}$ homeostasis in patient myoblasts, specifically in the time to return to baseline through clearance of the $\left[\mathrm{Ca}^{2+}\right]_{\mathrm{c}}$ following signal- or injury-mediated elevation, correlates with the increased ER fragmentation in the injured patient cells (Fig. 3c, d).

ER $\mathrm{Ca}^{2+}$ dysregulation causes ER stress, leading to muscle pathology ${ }^{42,43}$. However, we found that calnexin and GRP78 (molecular chaperones upregulated by ER stress) levels are similar in healthy and patient myoblasts (Fig. 5c, d) and treatment of myoblasts with the ER stress inducer thapsigargin (TG; $1 \mathrm{mM}$ TG for 0-24 h) caused a similar increase in calnexin and GRP78 levels in healthy and patient myoblasts (Fig. 5e-g). Thus, lack of ANO5 neither enhances ER stress nor alters the ability of the patient muscle cells to respond to ER stress.

\section{Efficient buffering of injury-triggered cytosolic $\mathrm{Ca}^{2+}$ increase improves patient myoblast repair}

Above results indicate that ANO5 deficiency dysregulates the clearance of injury-triggered elevation in $\left[\mathrm{Ca}^{2+}\right]_{\mathrm{C}}$, leading to increased ER fragmentation in injured cells. We thus hypothesized that it should be possible to correct this deficit by artificially buffering $\left[\mathrm{Ca}^{2+}\right]_{\mathrm{c}}$. To test this idea we loaded patient muscle cells with the cell permeant calcium chelator BAPTA-AM. The presence of BAPTA in the patient cell cytosol, corrected the rate at which the injury-triggered increase in $\left[\mathrm{Ca}^{2+}\right]_{\mathrm{c}}$ in the patient myoblasts returned to baseline (Fig. $6 \mathrm{a}-\mathrm{c}$ ) and also reduced the total $\left[\mathrm{Ca}^{2+}\right]_{\mathrm{c}}$ load (area under the curve for injurytriggered increase in Fluo-4 fluorescence) (Fig. 6b, d). We then examined ER fragmentation using RFP-KDEL in 


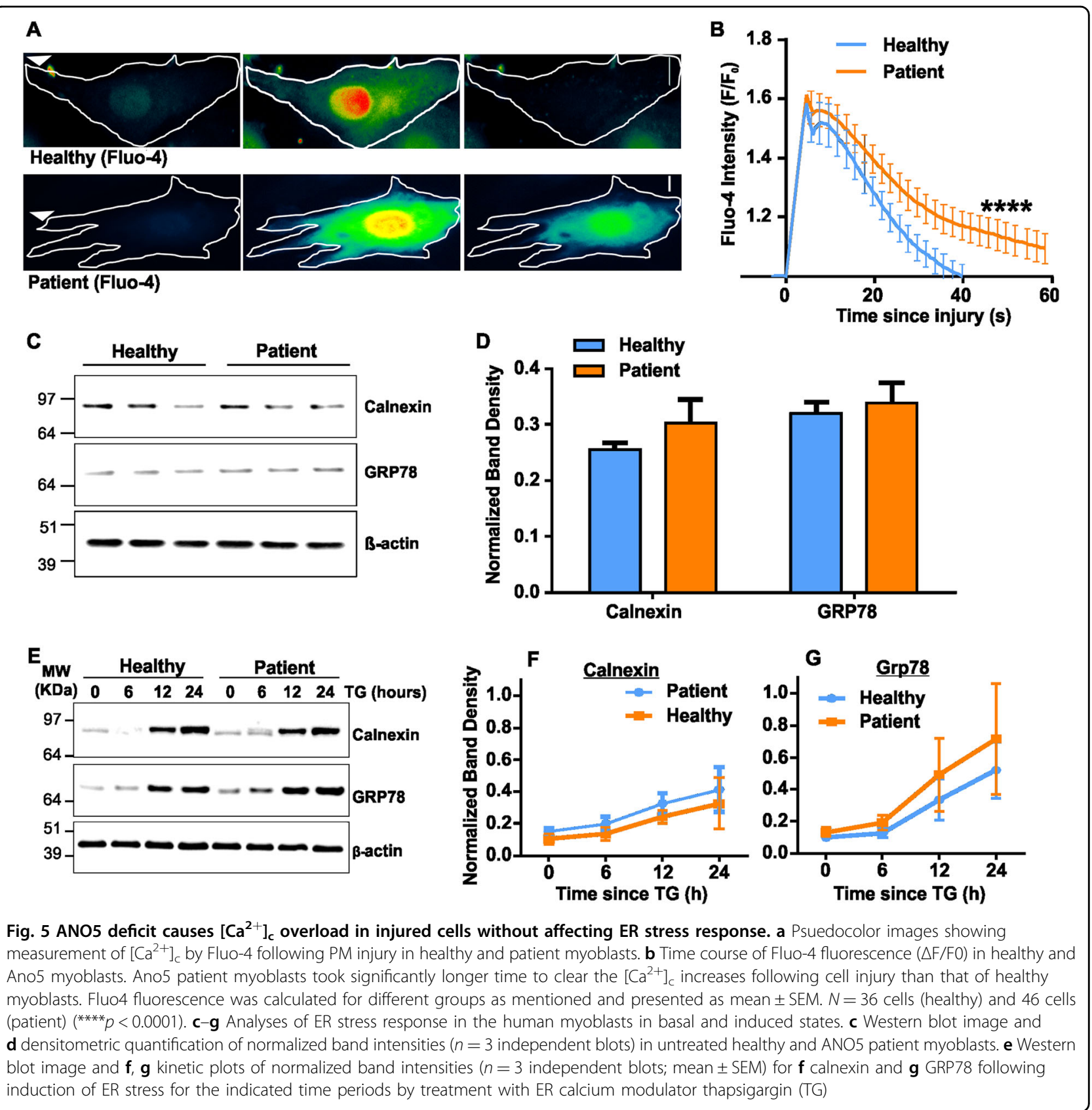

BAPTA-AM-treated patient myoblasts, which showed 2.5-fold lower ER fragmentation at the site of injury compared to vehicle-treated cells, making this comparable to the extent of ER fragmentation in healthy myoblasts (Fig. 6e, f).

Next, we tested if BAPTA-AM-treatment affects PMR kinetics. We found that BAPTA-AM treatment reversed the excessive FM1-43 dye entry following focal laser injury of plasma membrane of patient myoblasts (Fig. 6g, h). This reversal of the excessive dye entry in injured patient myoblasts enabled patient cells to repair in numbers that are comparable to the healthy human myoblasts (Fig. 6i). These data link several features of ANO5 deficiency in myoblasts. Upon focal injury, the patient cells display poor PMR, increased ER fragmentation and dysregulated $\mathrm{Ca}^{2+}$ homeostasis, which we hypothesize results in the poor PMR.

\section{Discussion}

We present the first human muscle cell model to examine the cellular function of ANO5 in muscular dystrophy. We generated myoblasts from a MMD3 patient with the c. $2272 \mathrm{C}>\mathrm{T}$ mutation in the ANO5 gene. This mutation corresponds to an $\mathrm{R} 758 \mathrm{C}$ substitution in the 


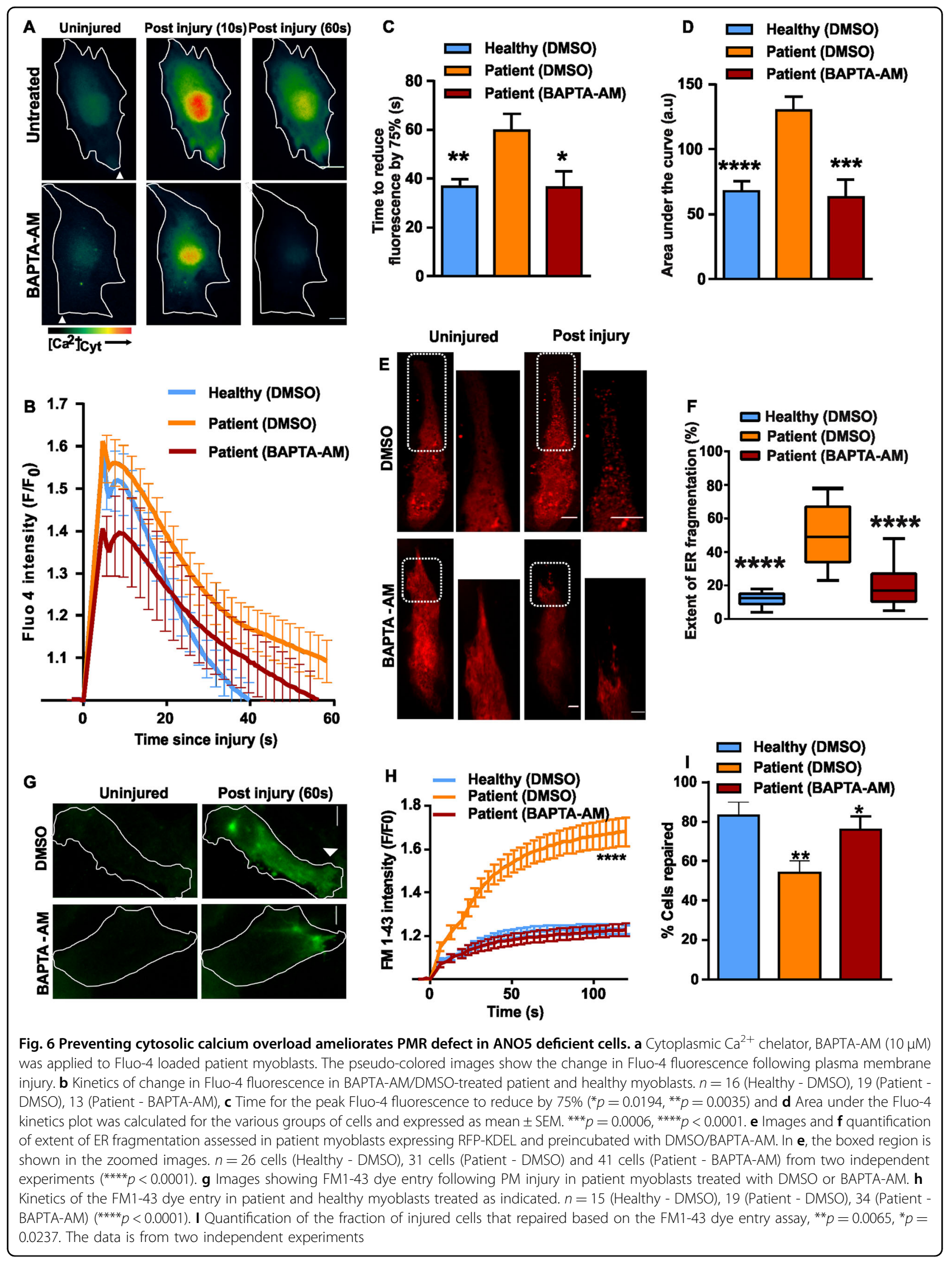


protein, causing it to be expressed at significantly reduced levels. Our 3D structural model of ANO5 places R758 in an ER-luminal loop between TM9 and TM10 (Fig. 4b) where it may play a role in establishing the dimerization interface. The R758C substitution may destabilize the protein, accounting for its low steady state level. Further work will be needed to clarify this point.

Using patient-derived myoblasts we find that while ANO5 is required for PMR, it is not required for myoblast fusion and myogenesis as has been reported for an ANO5-deficient mouse model ${ }^{26,28}$. While this difference between the mouse and human cells could reflect a species-specific myogenic role of ANO5, unrelated ANO5 knockout mouse models also show no developmental or regenerative myogenic deficit ${ }^{19,35}$. Furthermore, patients with ANO5 mutations do not exhibit developmental myogenic deficit. Thus, our results do not support a myogenic role of ANO5 in humans, but, just as in mouse muscles ${ }^{28}$, they demonstrate a requirement for ANO5 in human muscle cell sarcolemmal repair.

Unlike the sarcolemmal repair protein dysferlin, ANO5 plays a non-redundant role in sarcolemmal repair (Fig. 2). ANO5 expression fails to reverse the PMR defect in dysferlin deficient muscle fibers ${ }^{34}$ and we have shown that dysferlin enables PMR by facilitating lysosome docking and fusion ${ }^{33}$. ANO5-deficient patient fibroblasts have normal injury-triggered lysosome exocytosis ${ }^{29}$ and we find ANO5 deficit increases ER fragmentation following injury (Fig. 3). This implicates ANO5 in regulation of ER physiology. A TMEM16 protein family member Ist2 regulates ER morphology and ER-plasma membrane association in yeast ${ }^{44}$ while another member - ANO8 facilitates this in mammalian cells ${ }^{45}$. ER-PM tethering is crucial for cellular calcium homeostasis in mammalian cells $^{46}$. As ER fragmentation is linked to persistent increases in $\left[\mathrm{Ca}^{2+}\right]_{c}$, this observation further suggests a role for ANO5 in ER-mediated $\left[\mathrm{Ca}^{2+}\right]_{\mathrm{c}}$ homeostasis. Consistent with this we find that controlling injurytriggered $\left[\mathrm{Ca}^{2+}\right]_{\mathrm{c}}$ increase improves PMR in the patient cells. ANO5 is proposed to have both ion channel and phospholipid scramblase activities ${ }^{19,21,28}$, but this remains to be verified by direct assay of purified ANO5 in a reconstituted system. Interestingly, our 3D structural model of ANO5 places a number of disease-linked mutations close to the site at which activating $\mathrm{Ca}^{2+}$ ions bind the protein, and also in the vicinity of the SCRD and the lumenal entryway to the ion/lipid transport pathway (Fig. 4a, h). Our studies show that restoring the $\mathrm{Ca}^{2+}$ homeostasis is more important for the health of the patient muscle cells, while the relevance of the proposed scramblase function remains unclear.

The ER is the major intracellular $\mathrm{Ca}^{2+}$ store and the most likely organelle to normalize elevated $\left[\mathrm{Ca}^{2+}\right]_{\mathrm{C}}$ caused by plasma membrane injury. $\mathrm{Ca}^{2+}$ uptake across the ER is electrically neutral ${ }^{47}$, it must be accompanied by, for example, the expulsion of $\mathrm{K}^{+}$or $\mathrm{H}^{+}$ions from the ER and/or the import of $\mathrm{Cl}^{-}$into the ER lumen. The SERCA channel, important for excitation-contraction coupling in muscle, is proposed to accomplish electroneutrality by exchanging $\mathrm{Ca}^{2+}$ for $\mathrm{H}^{+}{ }^{48}$. However, it is unclear whether SERCA alone can handle the surge in $\left[\mathrm{Ca}^{2+}\right]_{\mathrm{c}}$ caused by injury and our data point to an important role for ANO5 in this process. Thus, the proposed channel activity of $\mathrm{ANO}^{22,26}$ could allow rapid electroneutral $\mathrm{Ca}^{2+}$ exchange through the counter-ion $\mathrm{Cl}^{-}$. This is consistent with the reported deficit in $\mathrm{ER} \mathrm{Ca}^{2+}$ influx due to poor $\mathrm{Cl}^{-}$transport across the ER membrane ${ }^{47}$.

In Summary, our data suggest that ANO5 influences cytosolic $\mathrm{Ca}^{2+}$ homeostasis by way of its ER-localized ion channel activity. This mode of action of ANO5 reveals a new membrane repair mechanism that depends on coping with the cytosolic $\mathrm{Ca}^{2+}$ increase that accompanies membrane injury. While other details of this process are subject of future studies, our finding that buffering of excess cytosolic $\mathrm{Ca}^{2+}$ improves repair of ANO5-patient muscle cells provides proof-of-principle for efficient removal of cytosolic $\mathrm{Ca}^{2+}$ in the muscle of ANO5 patients as a potential therapeutic strategy for treating LGMD2L and MMD3 patients.

\section{Methods \\ Human myoblasts}

Myoblast cultures were generated from muscle biopsy obtained from following ethical approval by Helsinki university human subject ethics committee and informed consent by the donor. The patient cells were obtained from the quadriceps of a 46-year-old Caucasian male with confirmed homozygous C.2272>T mutation in the ANO5 gene. The patient had disease symptoms since prior to the age of 25 years old, which includes elevated serum CK levels $(12,000-16,000 \mathrm{U} / \mathrm{L})$. The healthy cells were obtained from semi-tendonitis of a 25 year old Caucasian male donor with no known muscle disease or damage. All human samples were obtained with informed consent from each participant. Primary myoblasts were purified by magnetic activated cell sorting using anti-Cluster Designate 56 (CD 56 - a specific marker of myoblasts) coated beads (MACS, Miltenyl Biotech). Immortalization was performed using human telomerase reverse transcriptase (hTERT, to preserve telomere length) and cyclindependent kinase-4 (cdk-4, to overcome the p16 pathway) transgenes, as described previously ${ }^{49}$. The cells were cultured in Skeletal Muscle Cell Basal Media (PromoCell $\mathrm{GmbH}$, Heidelberg, Germany) containing $15 \%$ fetal bovine serum, $5 \%$ fetal calf serum and necessary supplements (hEGF, hbFGF, fetuin, insulin and dexamethasone). Hundred percent confluent myoblasts cultures were differentiated using Skeletal Muscle Cell Differentiation 
Medium (PromoCell GmbH, Heidelberg, Germany) with the differentiation supplements, as described previously ${ }^{50}$. Transfections were performed in OPTI-MEM (Invitrogen) using Lipofectamine LTX for $4 \mathrm{~h}$ followed by addition of full growth media and growing the cells for 24-48 h. LGMD2L fibroblasts were used, as described previously ${ }^{29}$. All the cell culture experiments were repeated using at least three independent cell cultures or as indicated in the figure legends.

\section{Injury assays}

These assays were performed as described previously $^{33,37}$.

\section{Glass bead injury}

Cells cultured on coverslips were transferred to cell imaging media (CIM) or phosphate buffered saline (PBS) (Sigma-Aldrich) containing $2 \mathrm{mg} / \mathrm{ml}$ of lysine-fixable FITC-dextran (Life Technologies). Cells were injured by rolling glass beads (Sigma-Aldrich) over the cells. They were allowed to heal at $371 \mathrm{C}$ for $5 \mathrm{~min}$, and then incubated at $37^{\circ} \mathrm{C}$ for $5 \mathrm{~min}$ in CIM/PBS buffer containing $2 \mathrm{mg} / \mathrm{ml}$ of lysine-fixable TRITC dextran (Life Technologies). Cells were fixed in 4\% PFA, and nuclei were counterstained with Hoechst 33342; the cells were then mounted in fluorescence mounting medium (Dako) and imaged as described in Supplemental Methods. The number of FITC-positive cells (injured and repaired) and TRITC-positive cells (injured and not repaired) were counted. The number of injured cells that failed to be repaired was expressed as a percentage of the total injured cells.

\section{Laser injury}

Cells cultured on coverslips were transferred to and incubated in CIM/PBS buffer with $1 \mathrm{mg} / \mathrm{ml}$ FM1-43 dye (Life Technologies) and placed in a Tokai Hit microscopy stage-top ZILCS incubator (Tokai Hit Co., Fujinomiyashi, Japan) maintained at $37^{\circ} \mathrm{C}$. For laser injury, a 1- to $5-\mu \mathrm{m}^{2}$ area was irradiated for $10 \mathrm{~ms}$ with a pulsed laser (Ablate!, 3i Intelligent Imaging Innovations, Inc. Denver, CO, USA). Cells were imaged at $2 \mathrm{~s}$ intervals using IX81 Olympus microscope (Olympus America, Center Valley, $\mathrm{PA}, \mathrm{USA}) . \mathrm{FM}$ dye intensity $(\triangle \mathrm{F} / \mathrm{F}$ where $\mathrm{F}$ is the original value) was used to quantify the kinetics of PMR and represented with intervals of five frames. For monitoring ER fragmentation, cells expressing ER-RFP or Sec61mCherry were imaged live by spinning disc confocal microscopy as the plasma membrane was injured focally by pulsed laser. The longest axis of the cell was drawn from the point of injury to the opposite end of the cell indicating the total length of the cell (Supplemental Fig. 6) and the length of portion of the cell where the tubular appearance of the ER was lost following injury and resulted in the punctate (bead-on-string) appearance was measured as the fragmented length of the cell (Supplemental Fig. 6). The percentage of the total length of the cell where injury caused the ER fragmentation was quantified for multiple cells and presented as mean $( \pm$ SEM $)$.

\section{Fluorescence recovery after photobleaching}

Immortalized myoblasts were grown on coverslips $24-48 \mathrm{~h}$ prior to imaging at upwards of $50 \%$ confluency at the time of imaging. Coverslips were mounted on holders and washed in Dulbecco's PBS (DPBS) and cells were imaged live using a 488-nm (for GFP) or 561-nm (for mCherry) lasers attached to the Hamamatsu spinning sisc confocal microscope on an Olympus IX81 stand. Cells were photobleached using the imaging laser and fluorescence recovery was monitored over time. Results were analyzed using Slidebook 6.0. The data for each condition were recorded, and recovery kinetics were analyzed and plotted.

\section{Immunocytochemistry}

Cells were fixed in 4\% PFA and blocked with 2\% BSA and $5 \%$ Goat serum in PBS for an hour at room temperature. Primary antibody incubations were followed by $1 \mathrm{~h}$ incubation with Alexa 488 or 594 goat secondary antibodies (Invitrogen, 1/1000) from the adequate species. Coverslips were mounted in Fluoromount G (Southern Biotech, Birmingham, USA) and examined through a Leica confocal microscope (TCS SP2.AOBS microscope, Leica, Germany) using the $488 \mathrm{~nm}$ and the $594 \mathrm{~nm}$ lines of an argon laser. The antibodies used for immuno-staining were Anti-ANO5 (NIH/UC Davis), Anti-MYH3 (Santacruz Biotechnology Inc).

\section{Western Blotting}

Cells were lysed with RIPA buffer (Sigma-Aldrich) containing protease inhibitor cocktail (Fisher Scientific, Waltham, MA, USA). Proteins transferred to nitrocellulose membranes were probed with the indicated antibodies against: Calnexin (Millipore), GRP78 (Santa Cruz) and $\beta$-actin (Santa Cruz). Primary antibodies were followed by the appropriate HRP-conjugated secondary antibodies (Sigma-Aldrich), and chemiluminescent western blotting substrate (Fisher, Waltham, MA, USA; GE Healthcare, Pittsburgh, PA, USA). The blots were then processed on Bio-Rad ChemiDoc Touch Imaging System (Bio-Rad).

\section{Quantitative RT PCR}

Real-time qRT-PCR was performed using total RNA $(1 \mu \mathrm{g})$ from subjected to DNAse I digestion $(1 \mathrm{U})$ in $1 \times$ DNAse I reaction buffer (Promega) by incubating at $37^{\circ} \mathrm{C}$ for $30 \mathrm{~min}$ to remove genomic DNA contamination. The 
reaction was inactivated by adding $1 \mu \mathrm{l}$ of stop solution (Promega) and heating for $10 \mathrm{~min}$ at $65^{\circ} \mathrm{C}$. Subsequently, the RNA sample was reverse transcribed to cDNA using Superscript II (Life Technologies) and oligo dT primers. The cDNA thus generated was amplified in triplicates in SYBR Green PCR Master Mix (Life Technologies) using $1 \mu \mathrm{M}$ of forward and reverse primers specific to each gene and $1 \mu \mathrm{l}$ of cDNA template in a total volume of $50 \mu \mathrm{l}$. Predesigned SYBR Green primer pairs specific to exon 1213 (Primer 1) and exons 16-17 (Primer 2) of human Anoctamin 5 gene (RefSeqID - NM_01142649) were obtained from Sigma Aldrich. Glyceraldehyde-3phosphate dehydrogenase (Gapdh) was used as internal control and the primers used were (forward) $5^{\prime}$ CCAGGAGCGAGACCCCACTAACA-3' and (reverse) 5'TCAAGTGGGCCCCGGCCTT-3'. The results were independently validated for both primers using 18srRNA as an independent loading control.

\section{$\mathrm{Ca}^{2+}$ measurement assays}

For measuring cytosolic $\mathrm{Ca}^{2+}$, myoblasts and fibroblasts cultured on coverslips were incubated with DMEM without FBS containing $10 \mu \mathrm{M}$ of Fluo-4-AM (Life technologies, USA) for $20 \mathrm{~min}$ at $37^{\circ} \mathrm{C}$ and $5 \% \mathrm{CO}_{2}$. After washing with prewarmed CIM, cells were submitted to laser injury as described before (ref). To monitor $\mathrm{Ca}^{2+}$ influx in the cell following the laser injury, cells were imaged following injury at an interval of 4-6 frames/s for the next $3 \mathrm{~min}$. For every image, the kinetic of $\mathrm{Ca}^{2+}$ influx was expressed by the amount of Fluo-4-AM that entered the cell by measuring the cellular Fluo-4-AM emission. For measuring the ER $\mathrm{Ca}^{2+}$, we used FRET based biosensor, T1ER ${ }^{51}$. Myoblasts were transfected with T1ER DNA $24 \mathrm{~h}$ before assay. $\mathrm{Ca}^{2+}$ influx into the cells was induced by $\mathrm{PM}$ injury in the presence of $2 \mathrm{mM} \mathrm{Ca}^{2+}$.

\section{Total internal reflection fluorescence (TIRF) microscopy}

TIRF was carried out on Olympus IX81 microscope equipped with Lambda DG-4 (Sutter Instruments, Novato CA) widefield illumination system and Evolve 512 EMCCD (Photometrics, Tucson, AZ) camera, with CellTIRFTM (Olympus) illuminator and $\times 60 / 1.45 \mathrm{NA}$ oil objective using Slidebook 6.0.15 (Intelligent Imaging Innovations, Inc. Denver, $\mathrm{CO}$ ). The cells being imaged were maintained at $37^{\circ} \mathrm{C}$ in the Tokai Hit microscope stage top ZILCS incubator (Tokai Hit Co., Japan). For TIRF imaging laser angle was set to obtain penetration depth of $70-120 \mathrm{~nm}$.

\section{Statistical analysis}

For membrane repair analysis the data are presented as averaged values for all of the myoblasts or myofibers used for that analysis. These averaged values were compared with each other using unpaired Student's t-test. For the densitometric analyses of the western blots, we performed unpaired $t$-test. For all the other experiments, when the data were not normally distributed or failed the equal variance test, the Mann-Whitney rank sum test in GraphPad Prism 6.0 (GraphPad Software, La Jolla, CA) was performed. Results of all the statistical significance testing for differences are indicated by the asterix symbol, while lack statistical significance is indicated by the absence of this symbol.

\section{Modeling ANO5 structure}

The protein sequence of human ANO5 (Swiss prot ID: Q75V66) was used to search the protein data bank using the HHPred server (https://toolkit.tuebingen.mpg.de/ \#/tools/hhpred) ${ }^{52}$. Three structurally related homologs were obtained: ANO10 (PDB ID 5OC9), ANO1 (PDB ID 5OYB) and nhTMEM16 (PDB ID 4WIS). The three structures are related in fold and have a root square mean deviation (RMSD) of $\sim 1 \AA$ between them. Since ANO5 contains a scramblase domain (Gyobu (2017) PNAS 114: 6274), further modeling was based on the relatively well characterized nhTMEM16 protein. Thus, the MODELLER server ${ }^{53}$ was used to generate a structural model of ANO5 based on nhTMEM16. The 3D structural model was energy minimized using the GROMOS 43B1 force field embedded in the Swiss PDB viewer tool ${ }^{54}$. The resulting monomer model was then used to generate a dimer model using the nhTMEM16 dimer as a template. The dimer model was energy minimized as above and used to locate residues important for lipid and ion transport and $\mathrm{Ca}^{2+}$ binding based on the sequence alignment with nhTMEM16. Selected disease linked mutations identified in ANO5 using the clinvar database ${ }^{21}$ (https://www.ncbi.nlm.nih.gov/clinvar) were mapped onto the structure. Jalview ${ }^{55}$ (http://www.jalview.org) was used to generate the figure showing alignment of ANO5 and nhTMEM16 (Fig. 4a and Supplemental Fig. 4) and USCF chimera ${ }^{56}$ was used to estimate RMSD and generate the figures showing the $3 \mathrm{D}$ structural models and residues of importance (Fig. 4b-h).

\section{Acknowledgements}

J.K.J. acknowledges support by the NIH grants (R01AR55686, U54HD090257).

\footnotetext{
Author details

${ }^{1}$ Center of Genetic Medicine Research, Children's National Health System, 111 Michigan Avenue, NW, Washington, DC 20010, USA. ${ }^{2}$ Center for Research in Myology, Sorbonne Universités, UPMC Université Paris 06, INSERM UMRS974, 47 Boulevard de l'hôpital, 75013 Paris, France. ${ }^{3}$ Department of Biochemistry, Weill Cornell Medical College, New York, NY 10065, USA. ${ }^{4}$ Department of Neurology, MRC Oulu, Oulu University Hospital and University of Oulu, Oulu, Finland. ${ }^{5}$ Department of Genomics and Precision Medicine, George Washington University, Washington, DC 20037, USA. ${ }^{6}$ MolBox LLC, 8115 Fenton Street \#304, Silver Spring, Maryland 20910, USA. ${ }^{7}$ Present address: Aix Marseille Université, UMR_S 910, Génétique Médicale et Génomique Fonctionnelle, 13385 Marseille, France
} 


\section{Conflict of interest}

The authors declare no conflict of interest.

\section{Publisher's note}

Springer Nature remains neutral with regard to jurisdictional claims in published maps and institutional affiliations.

The online version of this article (https://doi.org/10.1038/s41420-019-0197-z) contains supplementary material, which is available to authorized users.

Received: 28 May 2019 Revised: 10 June 2019 Accepted: 13 June 2019 Published online: 18 July 2019

\section{References}

1. Hicks, D. et al. A founder mutation in Anoctamin 5 is a major cause of limbgirdle muscular dystrophy. Brain: J. Neurol. 134, 171-182 (2011).

2. Sarkozy, A. et al. ANO5 gene analysis in a large cohort of patients with anoctaminopathy: confirmation of male prevalence and high occurrence of the common exon 5 gene mutation. Hum. Mutat. 34, 1111-1118 (2013).

3. Witting, N. et al. Anoctamin 5 muscular dystrophy in Denmark: prevalence, genotypes, phenotypes, cardiac findings, and muscle protein expression. J. Neurol. 260, 2084-2093 (2013).

4. Bolduc, V. et al. Recessive mutations in the putative calcium-activated chloride channel Anoctamin 5 cause proximal LGMD2L and distal MMD3 muscular dystrophies. Am. J. Hum. Genet. 86, 213-221 (2010).

5. Katoh, M. \& Katoh, M. GDD1 is identical to TMEM16E, a member of the TMEM16 family. Am. J. Hum. Genet. 75, 927-928 (2004).

6. Falzone, M. E., Malvezzi, M., Lee, B. C. \& Accardi, A. Known structures and unknown mechanisms of TMEM16 scramblases and channels. J. Gen. Physiol. 150, 933-947 (2018).

7. Whitlock, J. M. \& Hartzell, H. C. Anoctamins/TMEM16 proteins: chloride channels flirting with lipids and extracellular vesicles. Annu Rev. Physiol. 79, 119-143 (2017).

8. Alvadia, C. et al. Cryo-EM structures and functional characterization of the lipid scramblase TMEM16F. bioRxiv, 455261 (2018).

9. Kalienkova, V. et al. Stepwise activation mechanism of the scramblase nhTMEM16 revealed by cryo-EM. bioRxiv, 455287 (2018).

10. Watanabe, R., Sakuragi, T., Noji, H. \& Nagata, S. Single-molecule analysis of phospholipid scrambling by TMEM16F. Proc. Natl Acad. Sci. USA 115, 3066-3071 (2018).

11. Malvezzi, M. et al. Ca2+-dependent phospholipid scrambling by a reconstituted TMEM16 ion channel. Nat. Commun. 4, 2367 (2013).

12. Brunner, J. D., Lim, N. K., Schenck, S., Duerst, A. \& Dutzler, R. X-ray structure of a calcium-activated TMEM16 lipid scramblase. Nature 516, 207-212 (2014).

13. Brunner, J. D., Schenck, S. \& Dutzler, R. Structural basis for phospholipid scrambling in the TMEM16 family. Curr. Opin. Struct. Biol. 39, 61-70 (2016).

14. Lee, B. C. et al. Gating mechanism of the extracellular entry to the lipid pathway in a TMEM16 scramblase. Nat. Commun. 9, 3251 (2018).

15. Lee, B. C., Menon, A. K. \& Accardi, A. The nhTMEM16 scramblase is also a nonselective ion channel. Biophys. J. 111, 1919-1924 (2016).

16. Andra, K. K., Dorsey, S., Royer, C. A. \& Menon, A. K. Structural mapping of fluorescently-tagged, functional nhTMEM16 scramblase in a lipid bilayer. J. Biol. Chem. 293, 12248-12258 (2018).

17. Mizuta, K. et al. Molecular characterization of GDD1/TMEM16E, the gene product responsible for autosomal dominant gnathodiaphyseal dysplasia. Biochem. Biophys. Res. Commun. 357, 126-132 (2007).

18. Tsutsumi, S. et al. The novel gene encoding a putative transmembrane protein is mutated in gnathodiaphyseal dysplasia (GDD). Am. J. Hum. Genet 74, 1255-1261 (2004).

19. Gyobu, S. et al. A role of TMEM16E carrying a scrambling domain in sperm motility. Mol. Cell Biol. 36, 645-659 (2016).

20. Suzuki, J. et al. Calcium-dependent phospholipid scramblase activity of TMEM16 protein family members. J. Biol. Chem. 288, 13305-13316 (2013).
21. Gyobu, S., Ishihara, K., Suzuki, J., Segawa, K. \& Nagata, S. Characterization of the scrambling domain of the TMEM16 family. Proc. Natl Acad. Sci. USA 114, 6274-6279 (2017).

22. Di Zanni, E., Gradogna, A., Scholz-Starke, J. \& Boccaccio, A. Gain of function of TMEM16E/ANO5 scrambling activity caused by a mutation associated with gnathodiaphyseal dysplasia. Cell Mol. Life Sci. 75, 1657-1670 (2018).

23. Tian, Y., Schreiber, R. \& Kunzelmann, K. Anoctamins are a family of $\mathrm{Ca}^{2+}$ -activated Cl- channels. J. Cell Sci. 125, 4991-4998 (2012).

24. Duran, C., Qu, Z., Osunkoya, A. O., Cui, Y. \& Hartzell, H. C. ANOs 3-7 in the anoctamin/Tmem $16 \mathrm{Cl}$ - channel family are intracellular proteins. Am. J. Physiol. Cell Physiol. 302, C482-C493 (2012).

25. Tran, T. T. et al. TMEM16E (GDD1) exhibits protein instability and distinct characteristics in chloride channel/pore forming ability. J. Cell Physiol. 229, 181-190 (2014).

26. Whitlock, J. M., Yu, K., Cui, Y. Y. \& Hartzell, H. C. Anoctamin 5/TMEM16E facilitates muscle precursor cell fusion. J. Gen. Physiol. 150, 1498-1509 (2018).

27. Penttila, S. et al. Eight new mutations and the expanding phenotype variability in muscular dystrophy caused by ANO5. Neurology 78, 897-903 (2012).

28. Griffin, D. A. et al. Defective membrane fusion and repair in Anoctamin5deficient muscular dystrophy. Hum. Mol. Genet 25, 1900-1911 (2016).

29. Jaiswal, J. K. et al. Patients with a non-dysferlin Miyoshi myopathy have a novel membrane repair defect. Traffic 8, 77-88 (2007).

30. Mahjneh, I. et al. A new distal myopathy with mutation in anoctamin 5 . Neuromuscul. Disord. 20, 791-795 (2010).

31. Bansal, D. et al. Defective membrane repair in dysferlin-deficient muscular dystrophy. Nature 423, 168-172 (2003).

32. Bashir, R. et al. A gene related to Caenorhabditis elegans spermatogenesis factor fer-1 is mutated in limb-girdle muscular dystrophy type 2B. Nat. Genet. 20, 37-42 (1998).

33. Defour, A. et al. Dysferlin regulates cell membrane repair by facilitating injury-triggered acid sphingomyelinase secretion. Cell Death Dis. 5, e1306 (2014).

34. Monjaret, F. et al. The phenotype of dysferlin-deficient mice is not rescued by adeno-associated virus-mediated transfer of anoctamin 5. Hum. Gene Ther. Clin. Dev. 24, 65-76 (2013).

35. $\mathrm{Xu}$, J. et al. Genetic disruption of Ano5 in mice does not recapitulate human ANO5-deficient muscular dystrophy. Skelet. Muscle 5, 43 (2015).

36. Sui, T. et al. Development of muscular dystrophy in a CRISPR-engineered mutant rabbit model with frame-disrupting ANO5 mutations. Cell Death Dis. $\mathbf{9}$, 609 (2018).

37. Defour, A., Sreetama, S. C. \& Jaiswal, J. K. Imaging cell membrane injury and subcellular processes involved in repair. J Vis Exp. https://doi.org/10.3791/ 51106 (2014).

38. Subramanian, K. \& Meyer, T. Calcium-induced restructuring of nuclear envelope and endoplasmic reticulum calcium stores. Cell 89, 963-971 (1997).

39. Neussert, R., Muller, C., Milenkovic, V. M. \& Strauss, O. The presence of bestrophin-1 modulates the $\mathrm{Ca} 2+$ recruitment from $\mathrm{Ca} 2+$ stores in the ER. Pflug. Arch.: Eur. J. Physiol. 460, 163-175 (2010).

40. Jaiswal, J. K. \& Simon, S. M. Total internal reflection fluorescence microscopy for high-resolution imaging of cell-surface events. Curr. Protoc. Cell Biol. 4, 12 (2003). Unit 4

41. Sreetama, S. C., Takano, T., Nedergaard, M., Simon, S. M. \& Jaiswal, J. K. Injured astrocytes are repaired by Synaptotagmin XI-regulated lysosome exocytosis. Cell Death Differ. 23, 596-607 (2016).

42. Ikezoe, $\mathrm{K}$. et al. Dysferlin expression in tubular aggregates: their possible relationship to endoplasmic reticulum stress. Acta Neuropathol. 105, 603-609 (2003).

43. Ikezoe, K. et al. Endoplasmic reticulum stress in myotonic dystrophy type 1 muscle. Acta Neuropathol. 114, 527-535 (2007).

44. Manford, A. G., Stefan, C. J., Yuan, H. L., MacGurn, J. A. \& Emr, S. D. ER-to-plasma membrane tethering proteins regulate cell signaling and ER morphology. Dev. Cell 23, 1129-1140 (2012).

45. Jha, A. et al. Anoctamin 8 tethers endoplasmic reticulum and plasma membrane for assembly of $\mathrm{Ca}(2+)$ signaling complexes at the ER/PM compartment. EMBO J. https:/doi.org/10.15252/embj.2018101452 (2019).

46. Putney, J. W. Intimate plasma membrane-ER interactions underlie capacitative calcium entry:"Kissin'Cousins". Cell 99, 5-8 (1999).

47. Pollock, N. S., Kargacin, M. E. \& Kargacin, G. J. Chloride channel blockers inhibit Ca2+uptake by the smooth muscle sarcoplasmic reticulum. Biophys. J. 75, 1759-1766 (1998). 
48. Møller, J. V., Olesen, C., Winther, A.M. L. \& Nissen, P. The sarcoplasmic Ca 2 +-ATPase: design of a perfect chemi-osmotic pump. Q. Rev. Biophys. 43, 501-566 (2010).

49. Mamchaoui, K. et al. Immortalized pathological human myoblasts: towards a universal tool for the study of neuromuscular disorders. Skelet. Muscle 1, 34 (2011).

50. Kim, C. H. et al. Schisandrae fructus enhances myogenic differentiation and inhibits atrophy through protein synthesis in human myotubes. Int. J. Nanomed. 11, 2407-2415 (2016).

51. Bandara, S., Malmersjo, S. \& Meyer, T. Regulators of calcium homeostasis identified by inference of kinetic model parameters from live single cells perturbed by siRNA. Sci. Signal 6, ra56 (2013).
52. Zimmermann, L. et al. A completely reimplemented MPI bioinformatics toolkit with a new HHpred server at its core. J. Mol. Biol. 430, 2237-2243 (2018).

53. Webb, B. \& Sali, A. Comparative protein structure modeling using MODELLER. Curr. Protoc. Bioinf. 54, 56 1-5 637 (2016).

54. Schwede, T., Kopp, J., Guex, N. \& Peitsch, M. C. SWISS-MODEL: an automated protein homology-modeling server. Nucleic Acids Res. 31, 3381-3385 (2003).

55. Waterhouse, A. M., Procter, J. B., Martin, D. M., Clamp, M. \& Barton, G. J. Jalview Version 2-a multiple sequence alignment editor and analysis workbench. Bioinformatics 25, 1189-1191 (2009).

56. Pettersen, E. F. et al. UCSF Chimera-a visualization system for exploratory research and analysis. J. Comput. Chem. 25, 1605-1612 (2004). 